\title{
Resolution of a large infradiaphragmatic leak with endoscopic vacuum therapy after total gastrectomy
}

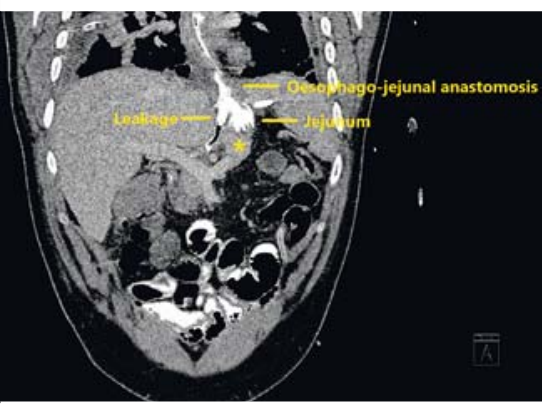

- Fig. 1 Computed tomography scan (Day 7 of admission, after third sponge replacement). The yellow lines highlight the leakage of the oral contrast at the level of the esophagojejunal anastomosis, which was still visible after the third sponge replacement. The tip of the sponge $\left({ }^{*}\right)$ inside the cavity is seen distal to the leak, between the left hepatic lobe and the body of the pancreas.

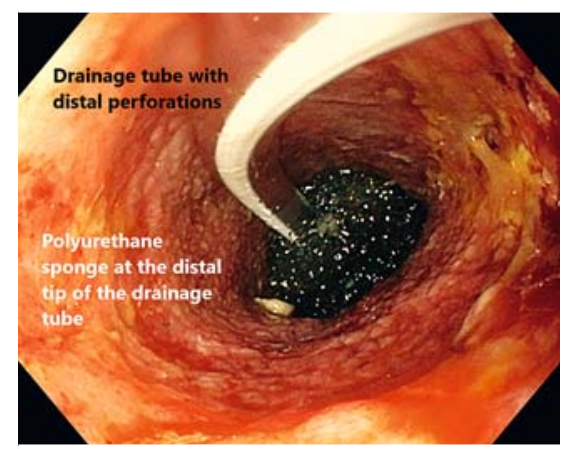

- Fig. 2 Placement of the Eso-SPONGE (B. Braun, Melsungen, Germany) in the distal part of the cavity.

Anastomotic insufficiency with leakage is a life-threatening complication after radical gastrectomy. Surgical reintervention may be associated with high rates of recurrence and increased morbidity [1]. Placement of a fully covered self-expandable metal stent is commonly performed as a first line conservative treatment. However, success rates after endoscopic stenting range between $63 \%$ and $91 \%$ [2]. Endoscopic vacuum therapy is a novel technique that allows continuous drainage of the leak, control of
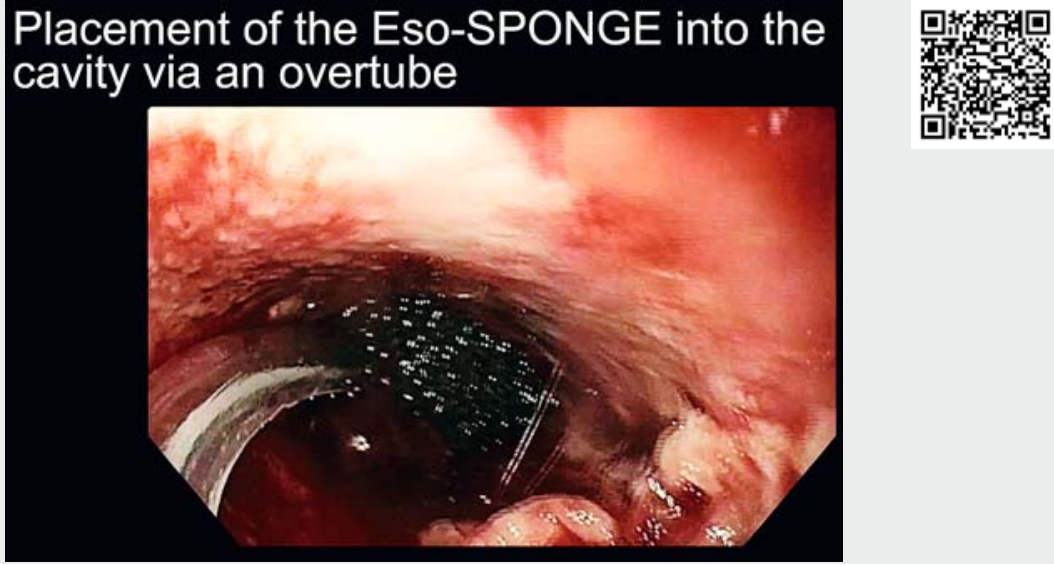

Video 1 Resolution of a large infradiaphragmatic leak with endoscopic vacuum therapy after total gastrectomy.

infection, and secondary wound healing process, and has shown high clinical success rates [3-5].

A 35-year-old man who underwent total gastrectomy and termino-lateral esophagojejunal anastomosis for gastric cancer at an outside hospital presented with an anastomotic leakage 5 days after surgery. Re-operation was unsuccessful. On postoperative Day 10, the patient was referred to our center with septic shock. The computed tomography (CT) scan confirmed the persistence of an anastomotic leak ( $>$ Fig. 1). An upper endoscopy showed a wall defect at the level of the anastomosis, which affected $30 \%$ of the circumference and gave access to a $10 \mathrm{~cm}$ (depth) $\times 5 \mathrm{~cm}$ (diameter) cavity with necrotic debris.

An Eso-SPONGE (B. Braun, Melsungen, Germany) was placed with endoscopic guidance inside the cavity and connected to a vacuum with a negative pressure of $100 \mathrm{mmHg}$ ( $\triangleright$ Video 1 ). The sponge was replaced $2-3$ times per week ( $>$ Fig.2). The septic shock quickly resolved with antibiotic therapy, and after 36 days of endoscopic treatment and 12 sponge replacements, the remaining wound cavity was $<2 \mathrm{~cm}$ (depth) $\times 1 \mathrm{~cm}$ (diameter) and the endoscopic therapy was discontinued. An upper endoscopy 10 days later ( $\mathbf{F i g . 3}$ ), and a CT scan and a barium swallow confirmed absence of leakage ( $>$ Fig.4, $\triangleright$ Fig.5). The patient was discharged 2 days later with good tolerance to an oral diet, and continued to do well at 3 months' follow-up.

Endoscopy_UCTN_Code_CPL_1AH_2AG

\section{Acknowledgments}

We thank Dr. Mario Pagés for his expertise and advice in selecting the radiologic pictures, and Dr. Andrés Cárdenas for proofreading and editing the text.

\section{Competing interests}

Drs. Sendino, Momblan, and de Lacy are speakers for B. Braun Company. 


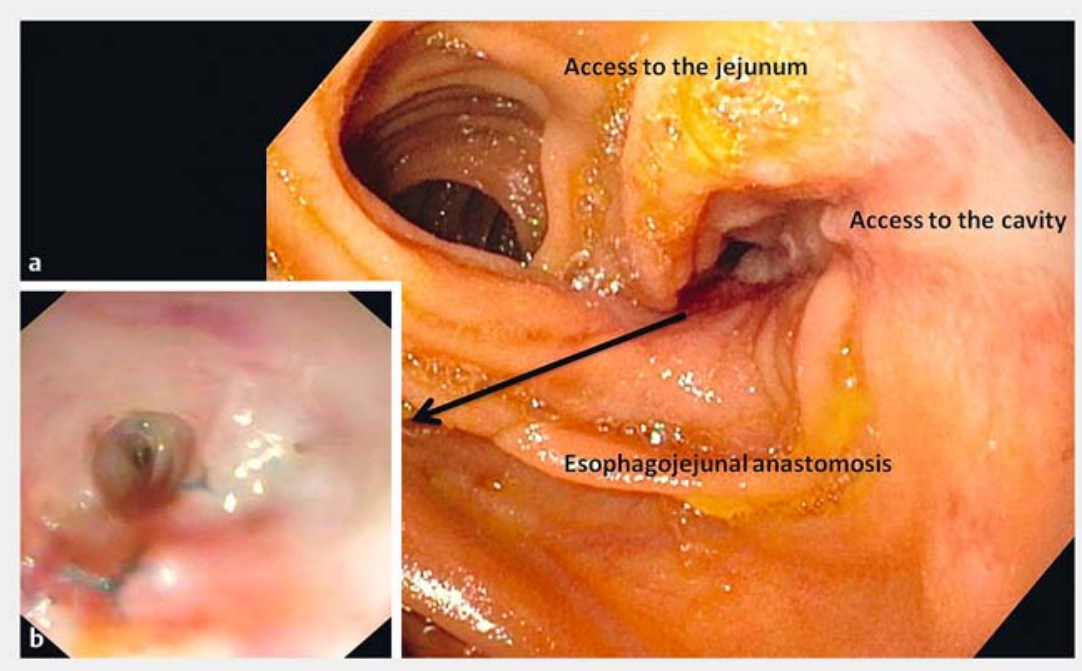

- Fig. 3 Upper endoscopy (10 days after the end of vacuum therapy). a The wall defect on the level of the anastomosis measured only $7 \mathrm{~mm}$ and was not accessible by a conventional gastroscope. b A pediatric gastroscope was used to access the cavity, which was only $2 \mathrm{~cm}$ long and had completely healed.

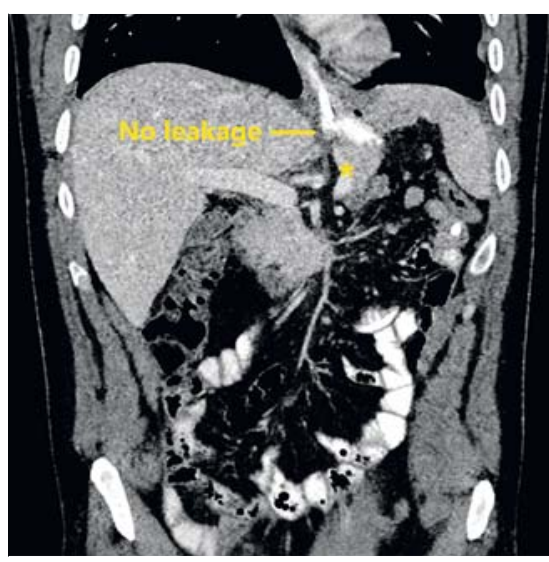

- Fig. 4 Computed tomography scan ( 10 days after the end of vacuum therapy). There was no evidence of contrast leakage and the cavity was completely resolved $\left({ }^{*}\right)$.

The authors

\section{Oriol Sendino ${ }^{1, "}$, Gerhard Jung ${ }^{2,}{ }^{*}$, Dulce} Momblan ${ }^{3}$, Antonio Maria de Lacy ${ }^{4}$

1 Endoscopy Unit, Gastroenterology Department, ICMDM, IDIBAPS, CIBEREHD, Hospital Clínic de Barcelona, University of Barcelona, Barcelona, Spain

2 Endoscopy Unit, Gastroenterology Department, ICMDM, Hospital Clínic de

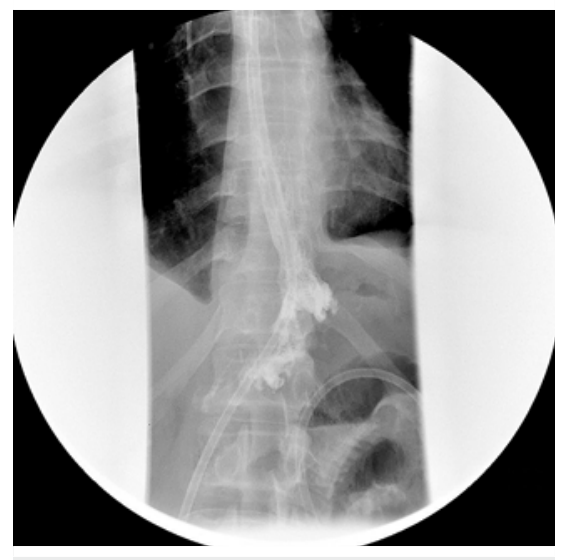

- Fig. 5 The barium swallow was performed 10 days after the end of therapy and there was no evidence of a leak.

Barcelona, University of Barcelona, Barcelona, Spain

3 Department of Gastrointestinal Surgery, ICMDM, Hospital Clínic de Barcelona, University of Barcelona, Barcelona, Spain

4 Department of Gastrointestinal Surgery, ICMDM, IDIBAPS, CIBEREHD, AIS Channel, Hospital Clínic de Barcelona, University of Barcelona, Barcelona, Spain
Corresponding author

\section{Oriol Sendino, MD, PhD}

Endoscopy Unit, Institute of Digestive Diseases and Metabolism, Hospital Clinic of Barcelona, Villarroel 170, Esc 3-2, 08036 Barcelona, Spain

Fax: +34-93-2279850

sendino@clinic.cat

\section{References}

[1] Messager M, Warlaumont M, Renaud F et al. Recent improvements in the management of esophageal anastomotic leak after surgery for cancer. Eur J Surg Oncol 2017; 43: $258-269$

[2] Persson S, Rouvelas I, Irino T et al. Outcomes following the main treatment options in patients with a leaking esophagus: a systematic literature review. Dis Esophagus 2017; 30: 1-10

[3] Hwang J], Jeong YS, Park YS et al. Comparison of endoscopic vacuum therapy and endoscopic stent implantation with self-expandable metal stent in treating postsurgical gastroesophageal leakage. Medicine (Baltimore) 2016; 95: e3416

[4] Laukoetter MG, Mennigen R, Neumann PA et al. Successful closure of defects in the upper gastrointestinal tract by endoscopic vacuum therapy (EVT): a prospective cohort study. Surg Endosc 2017; 31: 2687 -2696

[5] Rausa E, Asti E, Aiolfi A et al. Comparison of endoscopic vacuum therapy versus endoscopic stenting for esophageal leaks: systematic review and meta-analysis. Dis Esophagus 2018. doi:10.1093/dote/doy060

\section{Bibliography}

DOI https://doi.org/10.1055/a-0929-5508

Published online: 4.7.2019

Endoscopy 2019; 51: E376-E377

(c) Georg Thieme Verlag KG

Stuttgart · New York

ISSN 0013-726X

\footnotetext{
* These authors contributed equally to this work.
} 